\title{
Research Article \\ On the Implementation of a Dynamic Direction Modulation System with Vector Modulators
}

\author{
Edith Annette Cabrera-Hernández $\mathbb{D}^{1},{ }^{1}$ Josep Parron, ${ }^{1}$ and Alan Tennant ${ }^{2}$ \\ ${ }^{1}$ Department of Telecommunication and System Engineering, Escola d'Enginyeria, Universitat Autònoma de Barcelona (UAB), \\ Campus de la UAB, Q Building, Carrer de les Sitges, Bellaterra, Cerdanyola del Vallès, 08193 Barcelona, Spain \\ ${ }^{2}$ Department of Electronic \& Electrical Engineering, Portobello Centre, The University of Sheffield, Pitt Street, Sheffield (S1 4ET), UK
}

Correspondence should be addressed to Edith Annette Cabrera-Hernández; edithannette.cabrera@uab.cat

Received 26 November 2018; Revised 18 February 2019; Accepted 20 February 2019; Published 2 May 2019

Academic Editor: Shiwen Yang

Copyright ( 2019 Edith Annette Cabrera-Hernández et al. This is an open access article distributed under the Creative Commons Attribution License, which permits unrestricted use, distribution, and reproduction in any medium, provided the original work is properly cited.

\begin{abstract}
Dynamic directional modulation (DDM) has already proven to be an efficient technique to achieve physical layer security in wireless communications. System architectures based on vector modulators provide a flexible framework to implement synthesis methods that allow us to obtain increased security and/or independent multichannel transmissions. However, the implementation of DDM with vector modulators requires an accurate calibration (amplitude and phase) of every component in the RF path. In this contribution, we study the sensitivity of the response of a DDM system based on commercial vector modulators showing how to correct the nonideal behavior of all the components thanks to the flexibility provided by the vector modulator.
\end{abstract}

\section{Introduction}

Over the past few decades, wireless communication systems have expanded at an exponential rate, fueled by consumer demand and aided by increasing levels of technological advancement in terms of scalability, flexibility, and low reduced cost. However, wireless systems are more susceptible to interception than their wired counterparts due, partially, to the absence of physical boundaries in the transmission medium. An attractive solution to improve the security of wireless transmission is to apply encryption at the physical layer level to limit the amount of information that an illegitimate receiver can extract from the transmission medium [1].

The use of phased arrays to project nulls in the directions of illegitimate receivers [2] can be considered an antecedent to current physical layer security techniques. This method is simple and efficient but is limited by the need to know in advance the locations of illegitimate eavesdroppers that will tend to obfuscate their position.

Phased arrays can be also used to generate directional modulation (DM) [3-13]. This is an attractive option to achieve physical layer security since it is only necessary to know the direction of the legitimate receiver. A DM transmitter projects digitally modulated information signals into a prespecified secure spatial direction in free space, while simultaneously the constellation formats of the same signals are distorted in all other directions [3]. In the case that the distortion is dynamically updated (usually at the symbol rate) the DM is referred as dynamic (DDM) and provides better performance in terms of security than static DM (SDM) [4].

The hardware architectures of a DDM system with phased arrays mainly differ in the array feeding network implementation and we can distinguish: Fourier beamforming lens [5], radio frequency (RF) switch arrays [6-8], reconfigurable attenuators and/or phase shifters [9-11], and vector modulators $[3,12,13]$. In this work, we will consider the option of vector modulators (Figure 1) since it is very flexible and allows us to easily implement DM synthesis methods [3] to obtain increased security and/or independent multichannel transmissions $[12,13]$.

The performance of a DDM system is usually assessed in terms of the bit error rate (BER) increase of the received signal in other directions than the prespecified secure direction 


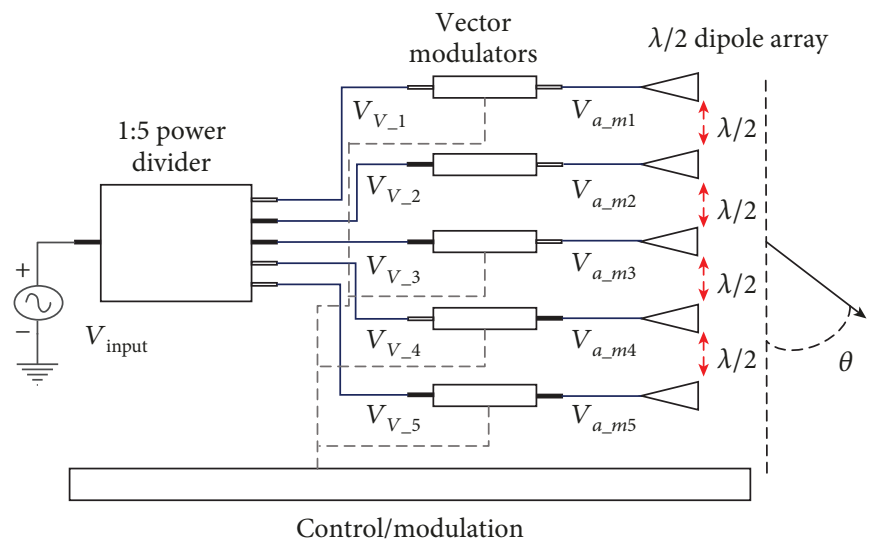

FiguRE 1: Transmission system demonstrator block diagram.
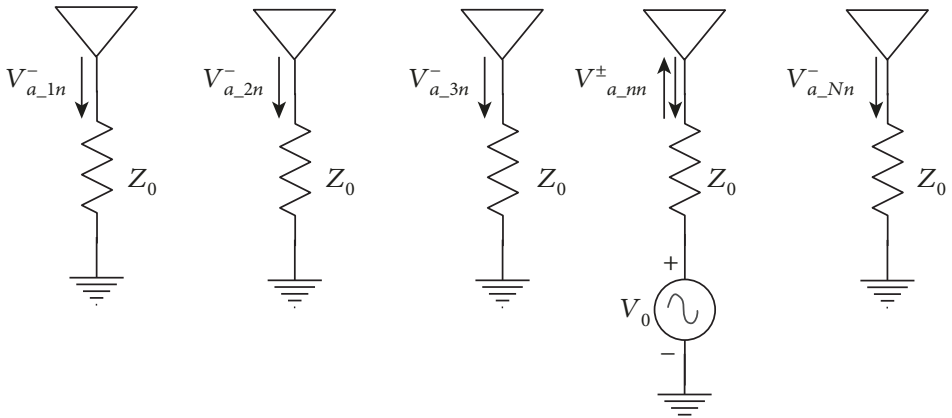

FiguRE 2: Geometry for obtaining the active element pattern of the element $n$ of the array of $N$ antennas.

[3-10] without considering how sensitive this BER is to small variations in the response of the components used. However, the implementation of DDM with vector modulators requires an accurate calibration (amplitude and phase) of every component in the RF path; otherwise, the transmitted constellations in the secure direction could be distorted as it was shown in a preliminary study in [14].

This contribution is aimed at studying the sensitivity of the response of a DDM system based on commercial vector modulators and showing how to correct the nonideal behavior of its components thanks to the flexibility provided by the vector modulator. The paper is organized as follows. Section 2 describes the system architecture and some concepts related to the generation of DDM. Section 3 derives the theoretical expressions for the $S_{21}$ parameter of the vector modulators for transmitting DDM considering real RF components. Section 4 evaluates the quality of the transmitted signal in the secure direction when commercial RF components are used and discusses the trade-off between power and security. Finally, the main conclusions of the work are summarized in Section 5.

\section{DDM System Description}

Figure 1 shows the scheme of the DDM transmission system under consideration. It is composed of a 5-way power divider, followed by 5 vector modulators (VM) that feed an

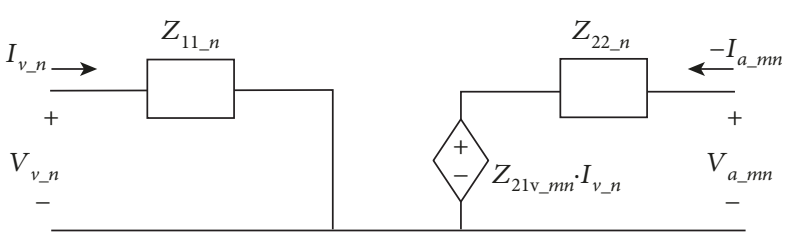

Figure 3: Equivalent circuit for $Z$ parameters of the vector modulator (unilateral assumption).

array of 5 half wavelength long dipole antennas in a side by side arrangement; the spacing between dipoles is $\lambda / 2(\lambda$ is the free space wavelength). The operating frequency of the RF source of the system is set arbitrarily to $2.45 \mathrm{GHz}$. A full model of the system has been created using the electromagnetic simulation software FEKO [15].

The array radiation pattern used to transmit symbol $m$ in a given direction $\theta$ can be expressed as

$$
S_{m}(\theta)=\sum_{n=1}^{5} B_{m n} \cdot \operatorname{AEP}_{n}(\theta),
$$

where $\operatorname{AEP}_{n}(\theta)$ is the active element pattern of antenna $n$ [16] and $B_{m n}$ is the weight of $\operatorname{AEP}_{n}(\theta)$ when transmitting symbol $m$. By varying the control inputs of the vector 


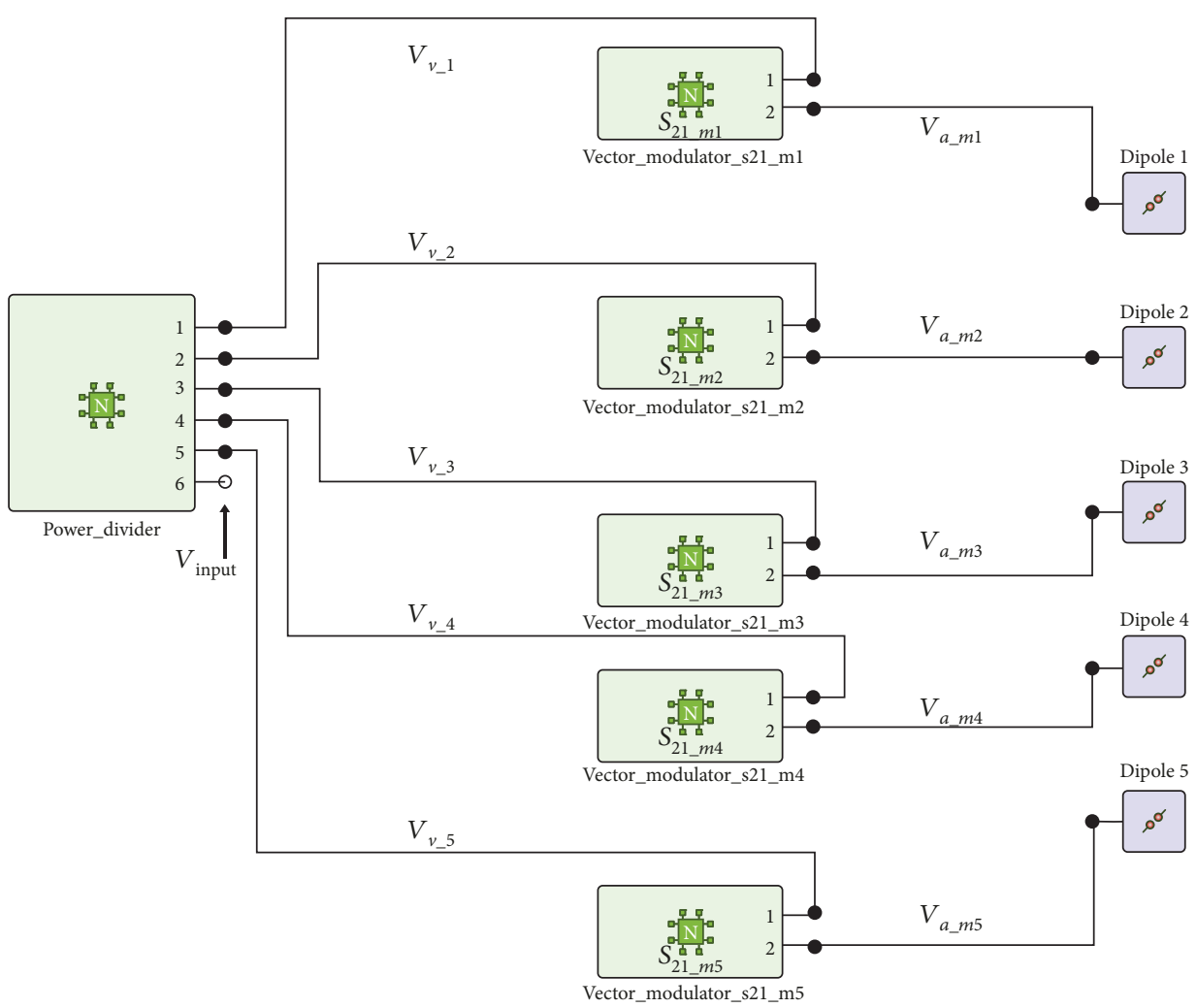

FIGURE 4: FEKO model of the transmission system demonstrator.

modulators, the parameter $S_{21}$ of each vector modulator can be adjusted to set the desired $B_{m n}$, thus allowing for tailored radiation patterns. As such, equation (1) is familiar as a classic form describing the array radiation pattern.

The required weights for a DM system $\left(B_{m n \_D M}\right)$ can be obtained using the orthogonal vector approach described in [3]: first, we design the weights for a nonDM (conventional) array $\left(B_{m n \_n o n D M}\right)$, and next, we add orthogonal noise $\left(W_{m n}\right)$ to the former weights $\left(B_{m n_{-} \mathrm{DM}}=B_{m n_{-} \text {nonDM }}+W_{m n}\right)$ in such a way as the constellation at the desired secure direction remains invariant. When $W_{m n}$ are dynamically updated (usually at the symbol rate), we are using DDM.

The directional modulation power efficiency $\left(\mathrm{PE}_{\mathrm{DM}}\right)$ is defined as the ratio of the power used in the non-DM array to the power used in the DM array [3]. It describes the extra power $\left(W_{m n}\right)$ injected into the array to obtain the directional modulation. In Section 4, it will be shown that decreasing $\mathrm{PE}_{\mathrm{DM}}$ increases the distortion in nondesired directions, thus improving the privacy of the communication. Therefore, there exists a compromise between security and $\mathrm{PE}_{\mathrm{DM}}$.

\section{Generation of the Radiation Patterns with the Vector Modulators}

This section is devoted to derive the expressions for the $S_{21}$ parameters of the vector modulators that will allow us to generate DDM with the scheme of Figure 1.
According to [16], $\operatorname{AEP}_{n}(\theta)$ can be obtained by feeding element $n$ of the array with all the other array elements terminated in matched loads $Z_{0}$, as shown in Figure 2.

With this scheme, the matrix that contains the input voltage of antenna $p$ when antenna $n$ is fed with a source of $1 \mathrm{~V}$ can be written as

$$
\left[V_{a_{-} p n}\right]=\frac{1}{2}\left([U]+\left[S_{a}\right]\right)
$$

where $\left[S_{a}\right]$ is the $S$ parameter matrix of the array and $[U]$ is the identity matrix of order $N$.

With the AEP of each antenna, we obtain the weights $B_{m n \text { DM }}$ by applying the procedure described in the previous section. Then, by using the superposition principle, the matrix that contains the input voltage in antenna $n$ when transmitting symbol $m$ is

$$
\left[V_{a_{-} m n}\right]=\frac{1}{2}\left([U]+\left[S_{a}\right]\right) \cdot\left[B_{m n_{-} \mathrm{DM}}\right]
$$

and the matrix that contains the input current in antenna $n$ when transmitting symbol $m$ becomes

$$
\left[I_{a \_m n}\right]=\frac{1}{2 Z_{0}}\left([U]-\left[S_{a}\right]\right) \cdot\left[B_{m n \_\mathrm{DM}}\right] .
$$

By combining (3) and (4), we can easily obtain the input impedance $\left(Z_{L_{-} m n}\right)$ and the corresponding reflection coefficient $\left(\Gamma_{L_{-} m n}\right)$. It must be noted that, even when each 


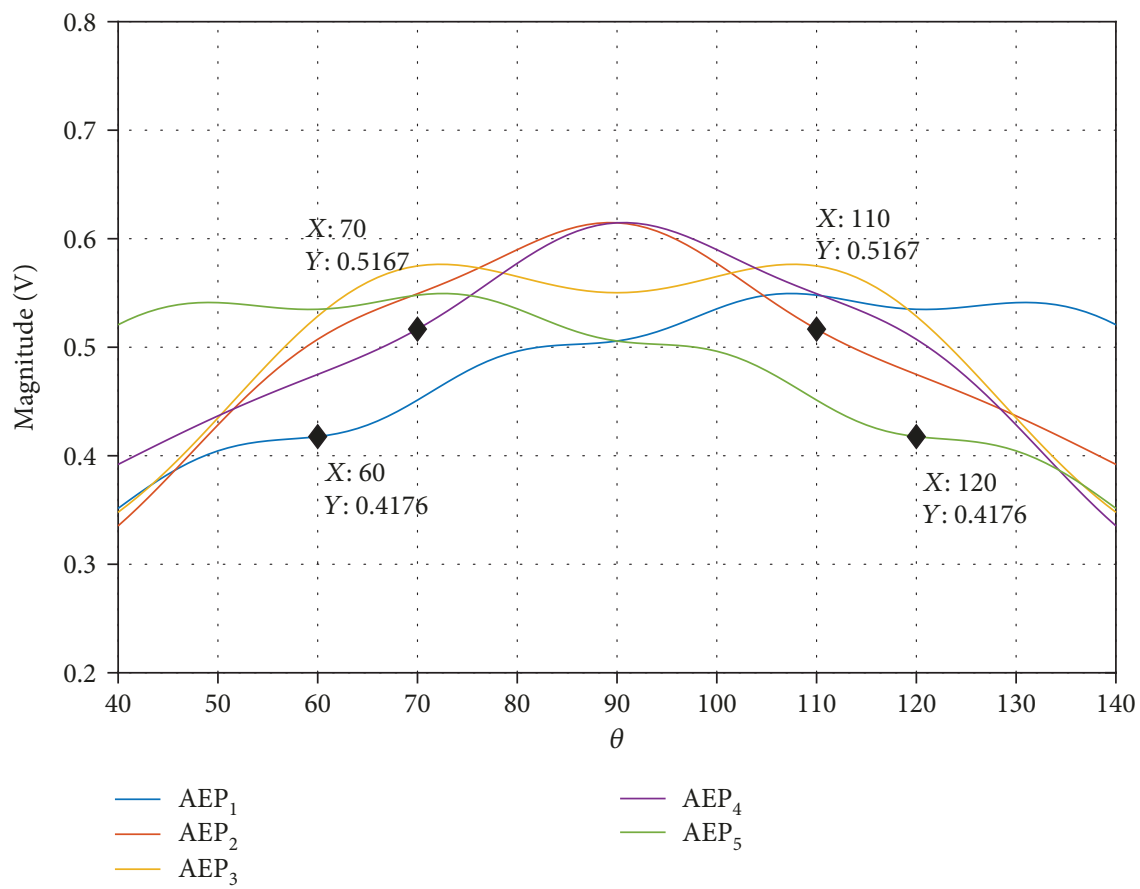

(a)

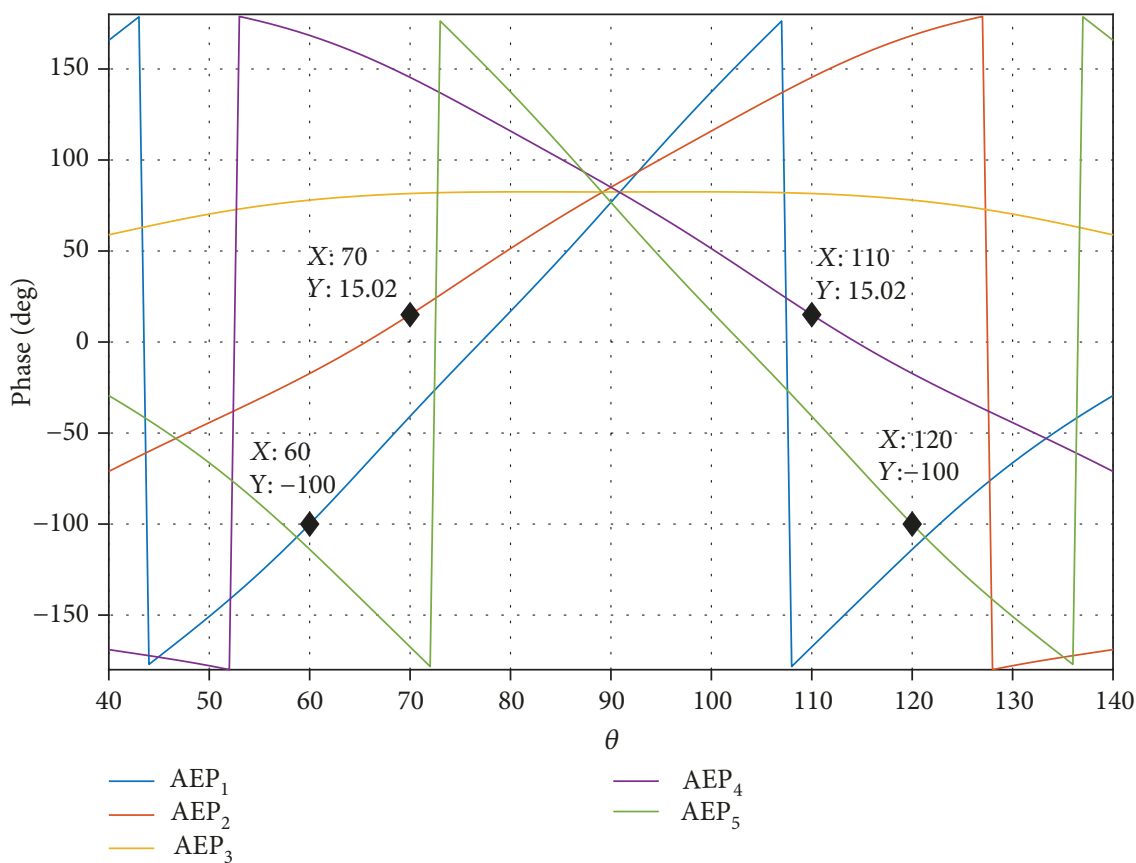

(b)

Figure 5: Active element pattern (H-plane) for an array of $5 \lambda / 2$ dipole antennas spaced $\lambda / 2$. (a) Magnitude. (b) Phase.

antenna is designed to be well matched (with all the other elements terminated in matched loads), $\Gamma_{L_{-} m n}$ changes with the element and transmitted symbol due to mutual coupling between the antennas.

It is also noted that for each element and symbol we also have

$$
B_{m n \_\mathrm{DM}}-V_{a \_m n}=Z_{0} I_{a \_m n} .
$$

Hence, it can be easily derived that

$$
\frac{B_{m n \_\mathrm{DM}}}{V_{a \_m n}}=\frac{2}{1+\Gamma_{L \_m n}} .
$$

Our goal now becomes setting the $S_{21}$ parameter for each vector modulator $n$ and symbol $m\left(S_{21 \_m n}\right)$ that generates the desired $V_{a_{-} m n}$ and $I_{a_{-} m n}$ at the antenna input. We are going to operate under the (realistic) assumptions 


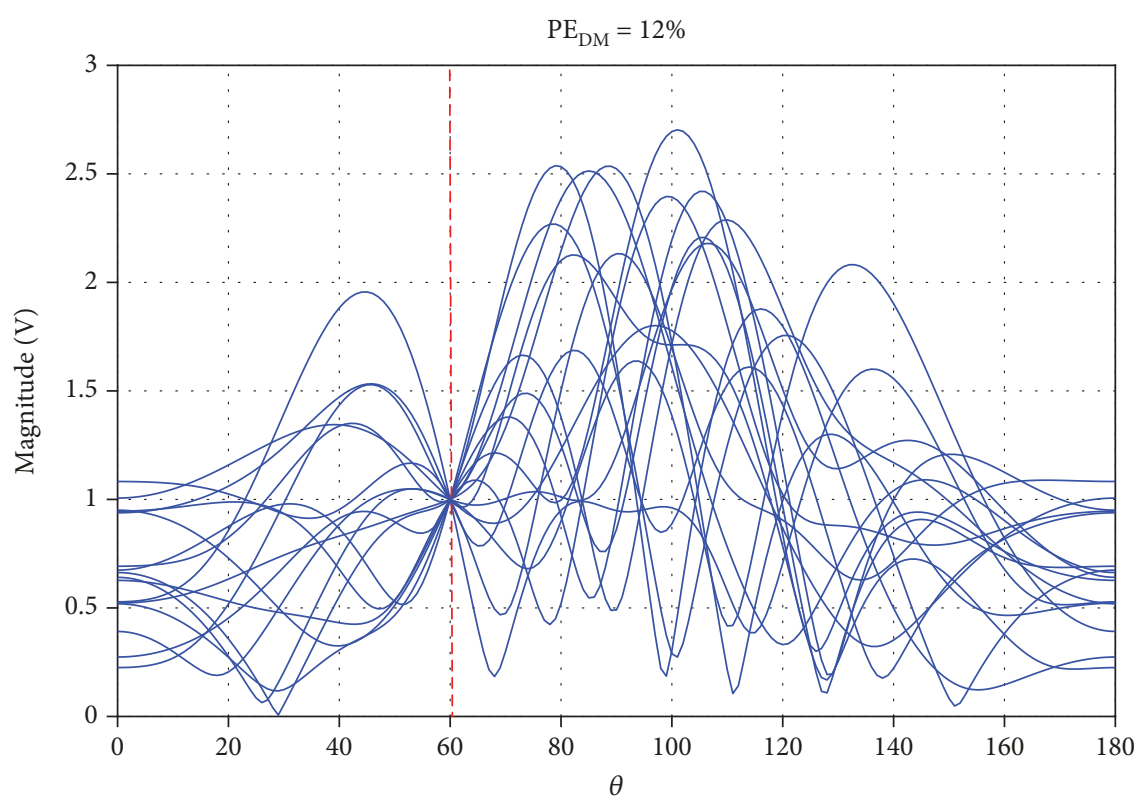

(a)

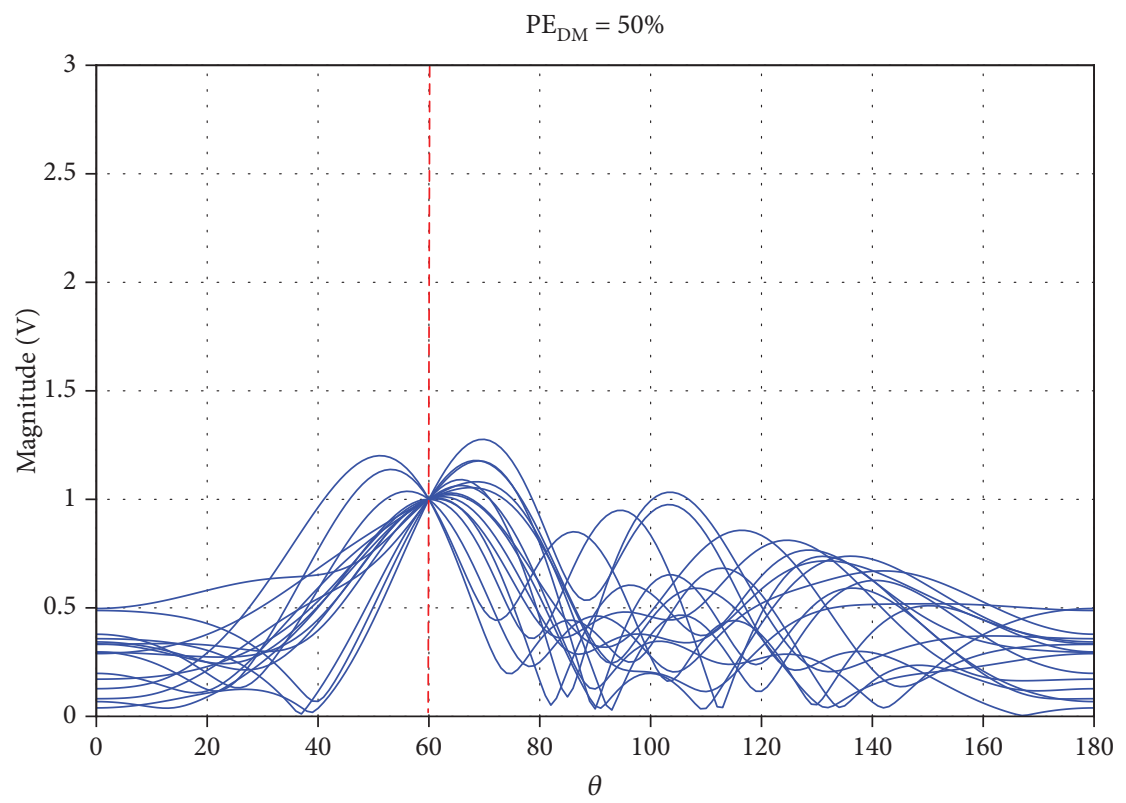

(b)

Figure 6: Magnitude of radiation patterns that transmit the same symbol at $60^{\circ}$ for two different values of $\mathrm{PE}_{\mathrm{DM}}$ (ideal power divider and vector modulator).

that the vector modulator is a unilateral network $\left(S_{12}=0\right)$, and $S_{11}$ and $S_{22}$ do no not change with the symbol to transmit.

The equivalent circuit for $Z$ parameters of the vector modulator is depicted in Figure 3. The $Z$ parameters are related to the $S$ parameters through the following equations that assume $S_{12}=0$ [17]:

$$
Z_{i i \_n}=Z_{0} \frac{\left(1+S_{i i \_n}\right)}{\left(1-S_{i i \_n}\right)}, \quad i=1,2,
$$

$$
Z_{21 \_m n}=2 Z_{0} \frac{S_{21 \_m n}}{\left(1-S_{11 \_n}\right)\left(1-S_{22 \_n}\right)}
$$

From Figure 3, it is straight forward that

$$
V_{a \_m n}-Z_{21 \_m n} \cdot I_{\nu \_n}=-Z_{22 \_n} \cdot I_{a \_m n}
$$

by substituting (7) and (8) into (9), and after a few mathematical manipulations, we obtain 


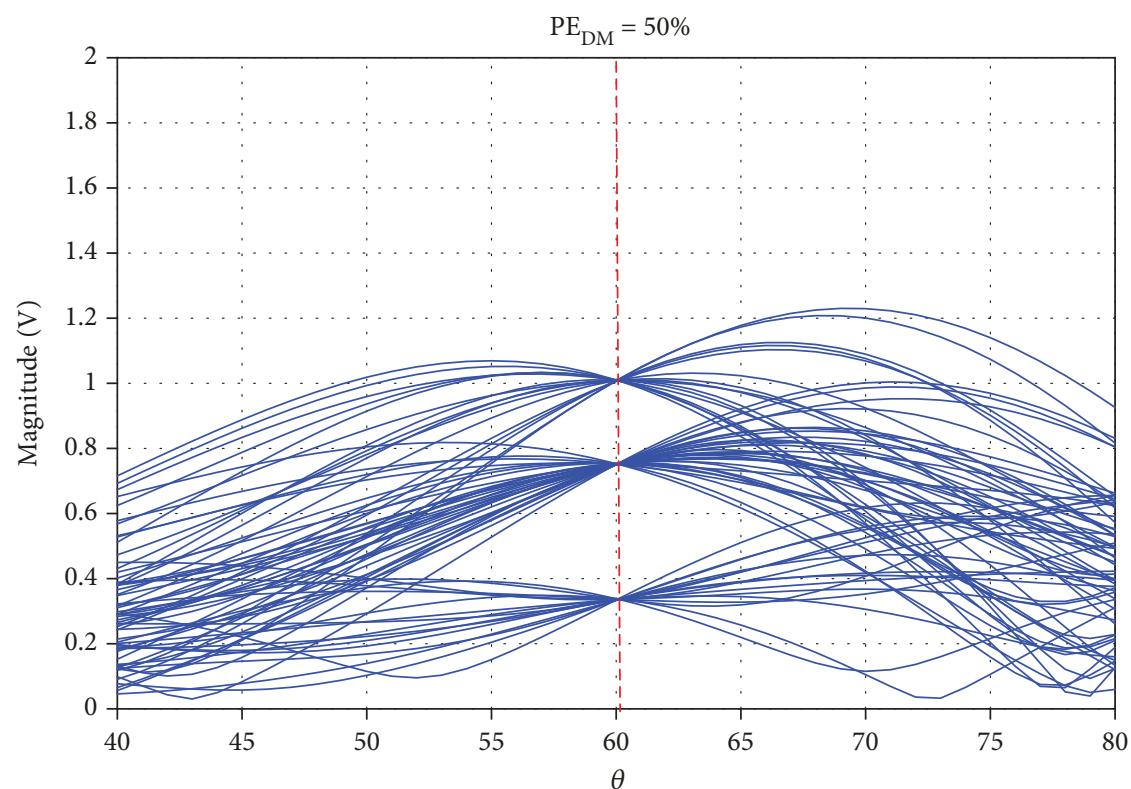

(a)

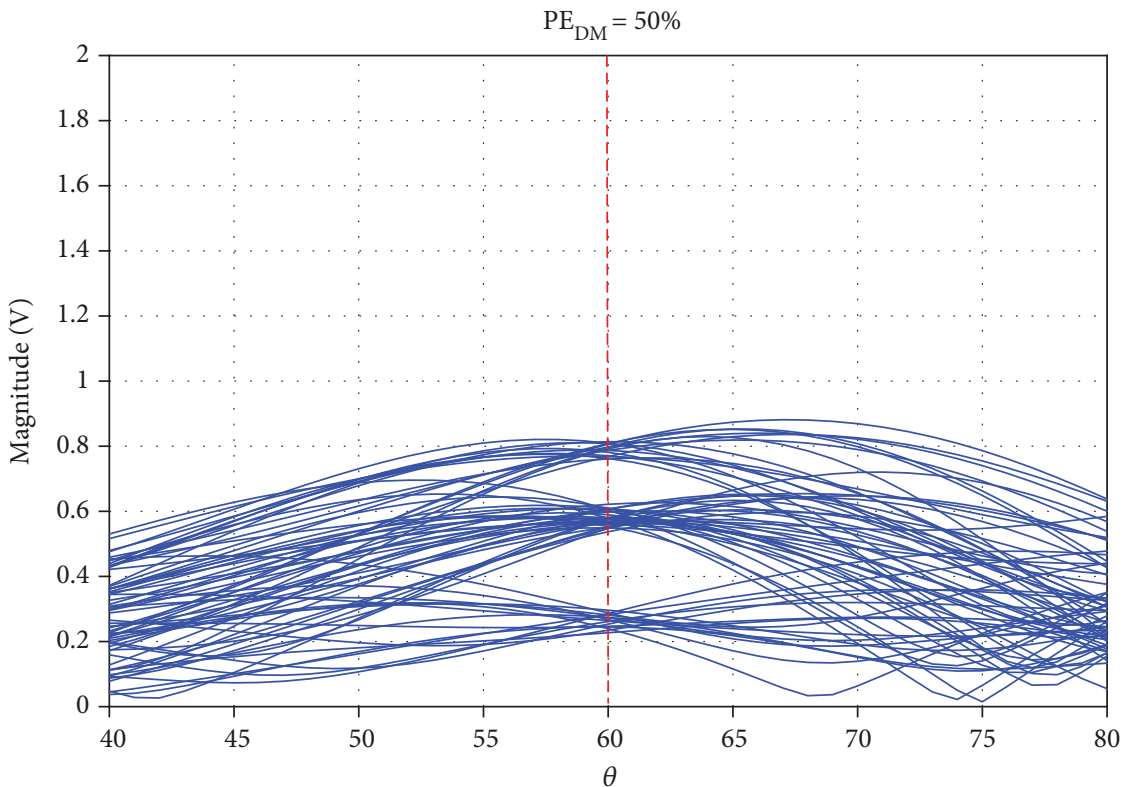

(b)

FIgURE 7: Magnitude of radiation patterns that transmit a 16-QAM constellation at $60^{\circ}\left(\mathrm{PE}_{\mathrm{DM}}=50 \%\right)$ using (12) to compute $S_{21 \_m n}$ in the vector modulators. (a) Ideal components. (b) Real components.

$$
S_{21 \_m n}=\frac{\left(1-S_{22 \_n} \Gamma_{L \_m n}\right)}{\left(1+\Gamma_{L \_m n}\right)}\left(1+S_{11 \_n}\right) \frac{V_{a \_m n}}{V_{v \_n}},
$$

where $V_{v \_n}$ is the input voltage for each vector modulator (which does not depend on the transmitted symbol). Finally, recalling (6), we obtain

$$
S_{21 \_m n}=\frac{\left(1-S_{22 \_n} \Gamma_{L \_m n}\right)}{2}\left(1+S_{11 \_n}\right) \frac{B_{m n \_D M}}{V_{v \_n}} .
$$

And for perfectly matched vector modulators $\left(S_{i i_{-} n}=0\right.$, $i=1,2$ ), (11) becomes

$$
S_{21 \_m n}=\frac{B_{m n \_D M}}{2 V_{v \_n}} .
$$

\section{Performance Assessment of a DDM System with Real Components}

Figure 4 shows the FEKO model of the DDM system based on vector modulators of Figure 1. The dipole array is evaluated through full electromagnetic simulation to include mutual coupling effects between antennas. Each component of the array feeding network is characterized by its respective 


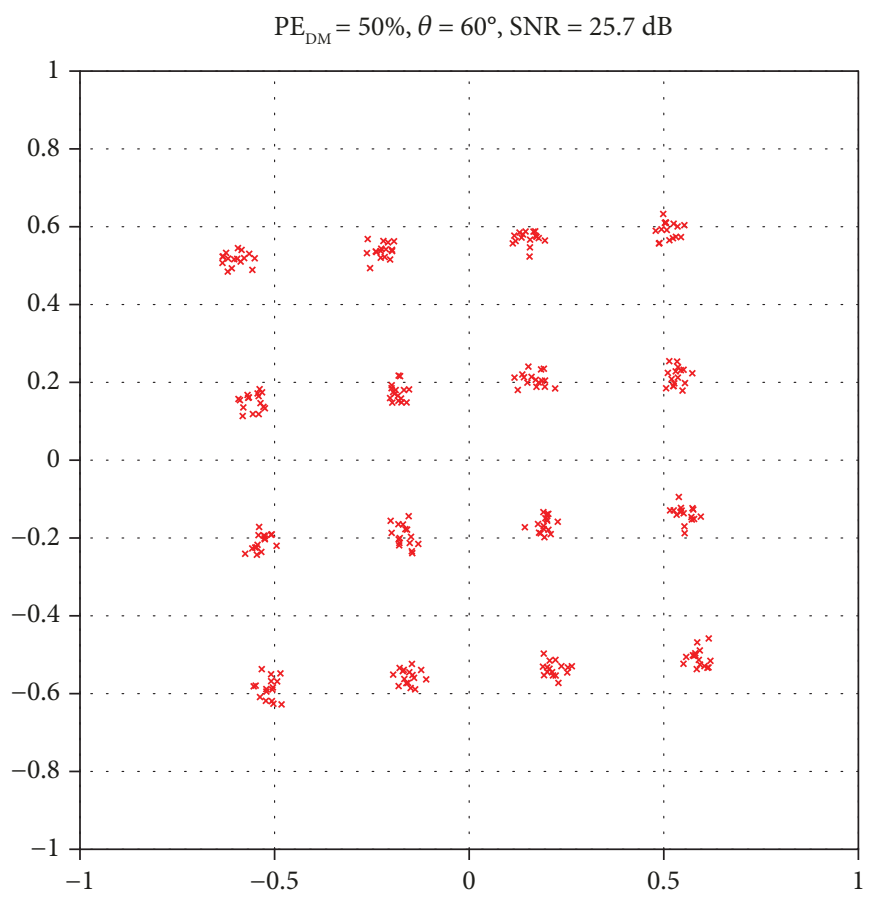

(a)

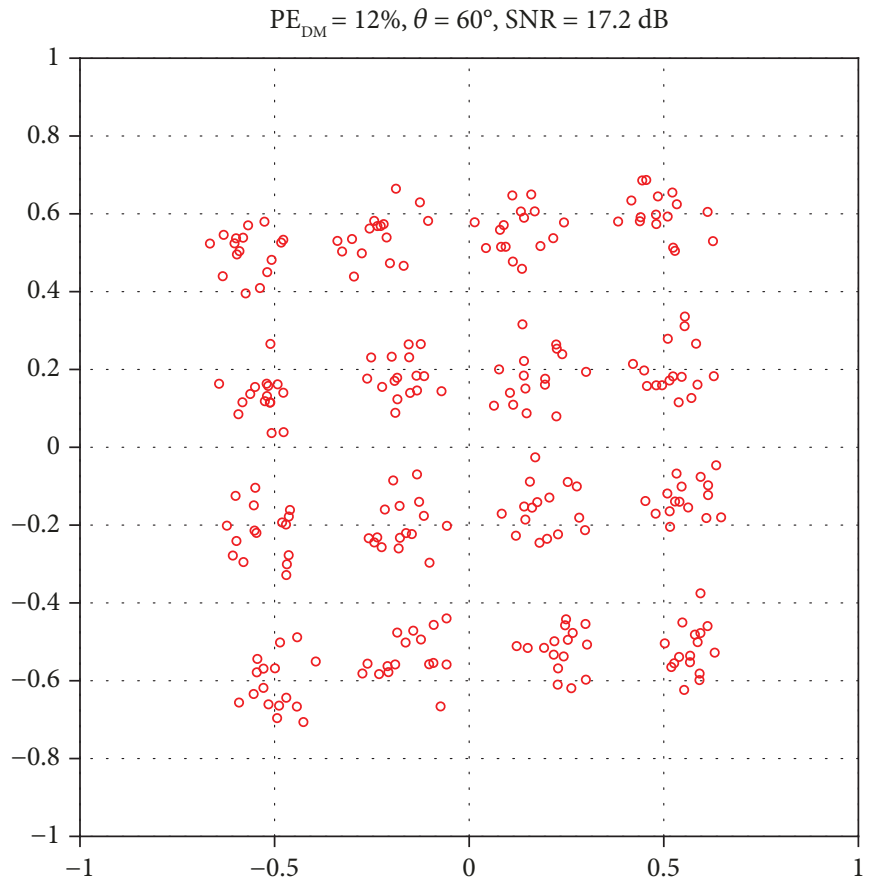

(b)

FIGURE 8: DDM 16-QAM modulation transmitted at the secure direction $60^{\circ}$ using real components and (12) to compute $S_{21 \_m n}$ in the vector modulators. (a) $\mathrm{PE}_{\mathrm{DM}}=50 \%$. (b) $\mathrm{PE}_{\mathrm{DM}}=12 \%$.

$S$ parameters. Then, once we compute $S_{21 \_m n}$, using either (11) or (12), we can obtain the radiation patterns for transmitting every symbol with very low computational requirements. In the first approach, we are not going to consider the transmission lines that connect the main components of our system (power divider, vector modulators, and antenna array) since their effects can be easily embedded inside the components.

In a previous step, the AEP of each dipole has been evaluated (Figure 5) and used to obtain the array weights $B_{m n}$ that will generate the desired radiation patterns for each symbol $m$. 


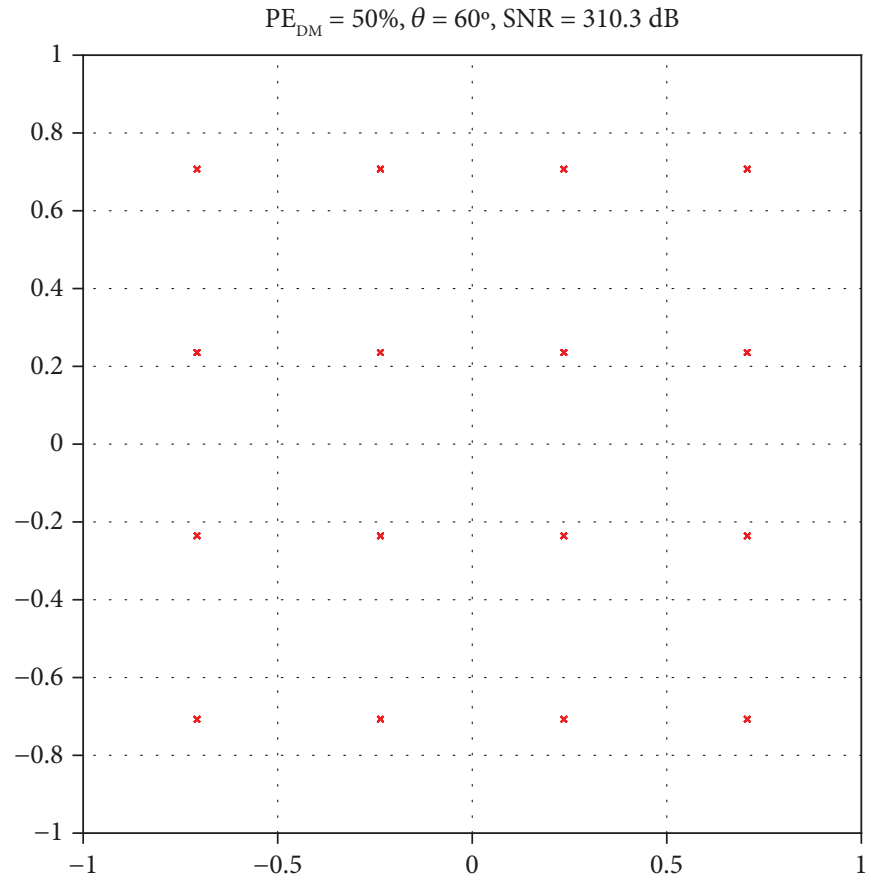

(a)

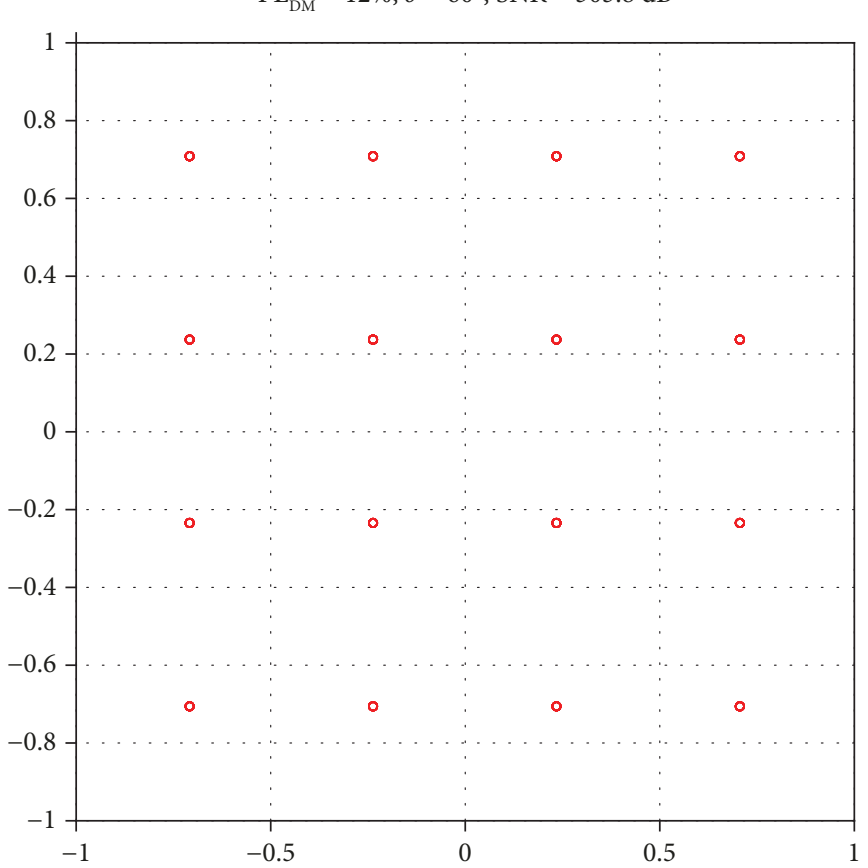

(b)

FIGURE 9: DDM 16-QAM modulation transmitted at the secure direction $60^{\circ}$ using real components and (11) to compute $S_{21 \_m n}$ in the vector modulators. (a) $\mathrm{PE}_{\mathrm{DM}}=50 \%$. (b) $\mathrm{PE}_{\mathrm{DM}}=12 \%$.

As a starting point, we use (12) to evaluate $S_{21 \_m n}$. We assume

(1) Ideal power divider: all ports perfectly matched, balanced power division (amplitude and phase), and isolated output ports
(2) Ideal vector modulators: both ports perfectly matched and $S_{12}=0$

Figure 6 shows simulated radiation patterns that can be used to transmit the same symbol in the secure direction of $60^{\circ}$ for two different values of $\mathrm{PE}_{\mathrm{DM}}$. In the case of ideal 


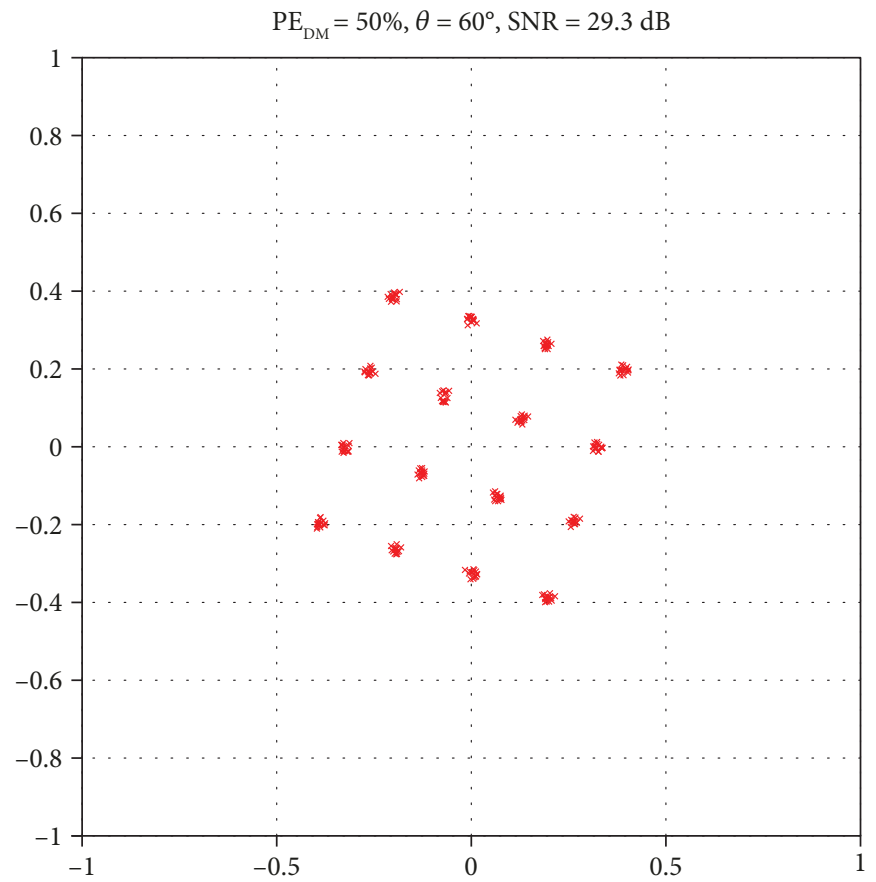

(a)

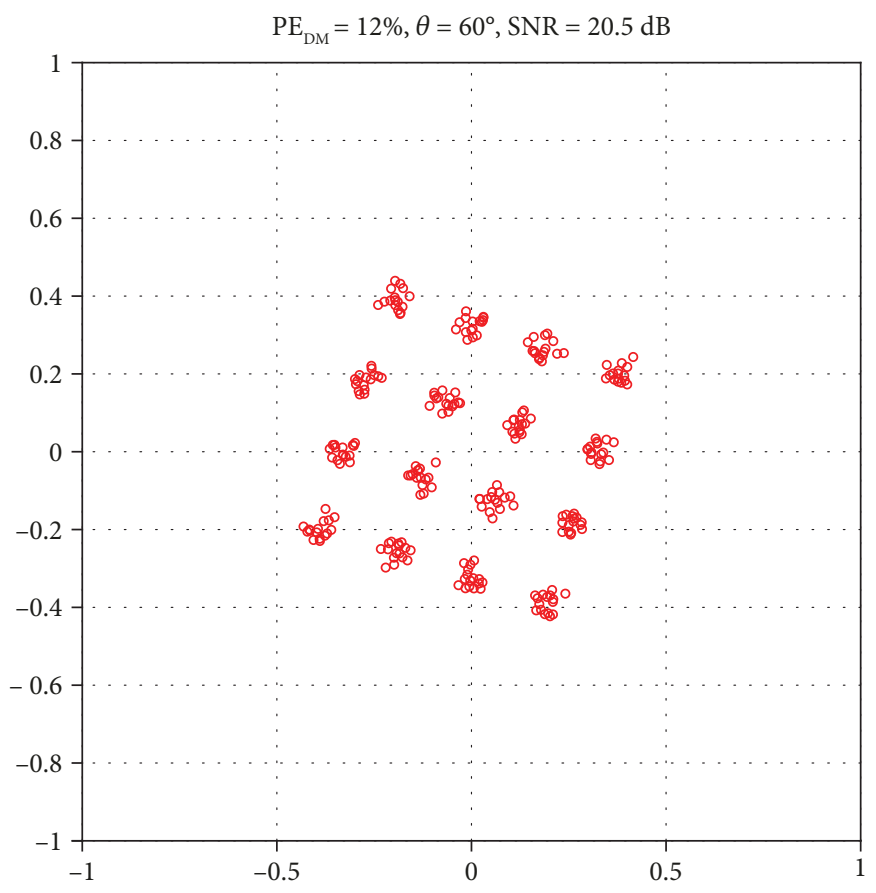

(b)

FiguRE 10: DDM 16-QAM modulation transmitted at the secure direction $60^{\circ}$ using real components, coaxial cables and (12) to compute $S_{21 \_m n}$ in the vector modulators; AEP measurement did not consider coaxial cables. (a) $\mathrm{PE}_{\mathrm{DM}}=50 \%$. (b) $\mathrm{PE}_{\mathrm{DM}}=12 \%$.

components, any constellation can be (theoretically) produced in a desired observation angle without issue.

In Figure 6, it is also important to highlight the trade-off between transmitted power and security: as $\mathrm{PE}_{\mathrm{DM}}$ decreases ( $W_{m n}$ power increases), we have a narrower secure beam width around $60^{\circ}$ and the distortion of symbols in undesired directions becomes larger.
Next, we introduce the characteristics of real components into the system. The power divider outputs will be assigned a nominal amplitude balance of $\pm 1.2 \mathrm{~dB}$ [18] and a phase variation of $\pm 3^{\circ}$. We also assume the vector modulators of [19] which show a measured $S_{12}$ parameter always below $-70 \mathrm{~dB}$ and input and output return losses around 9 and $10 \mathrm{~dB}$, respectively. 


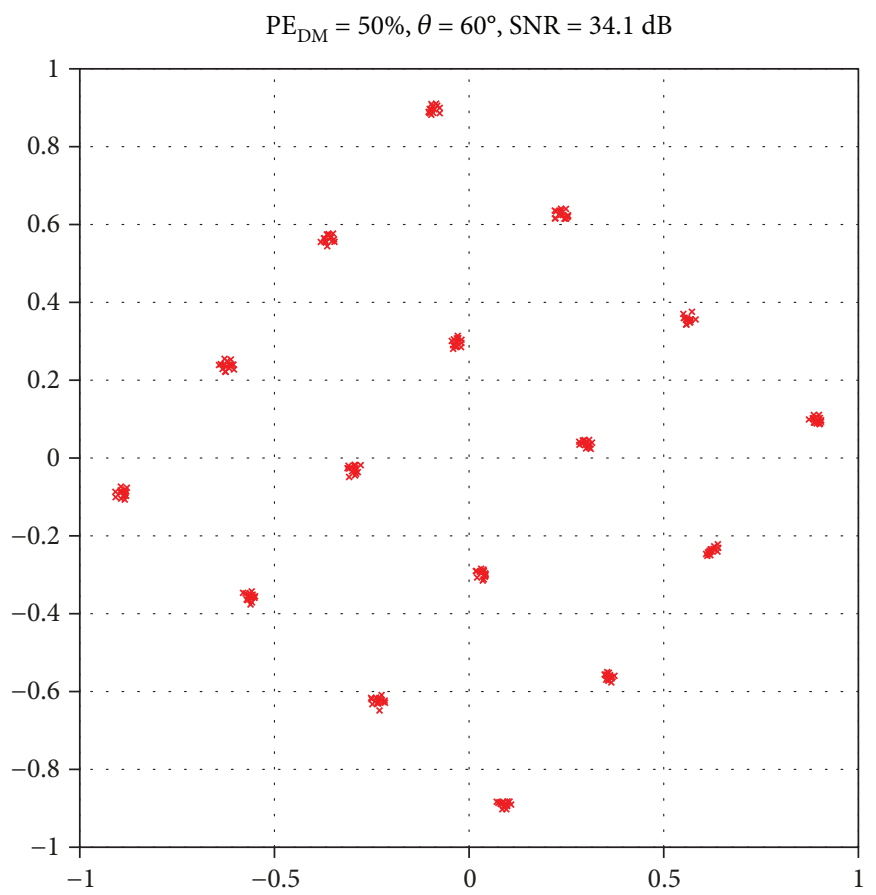

(a)

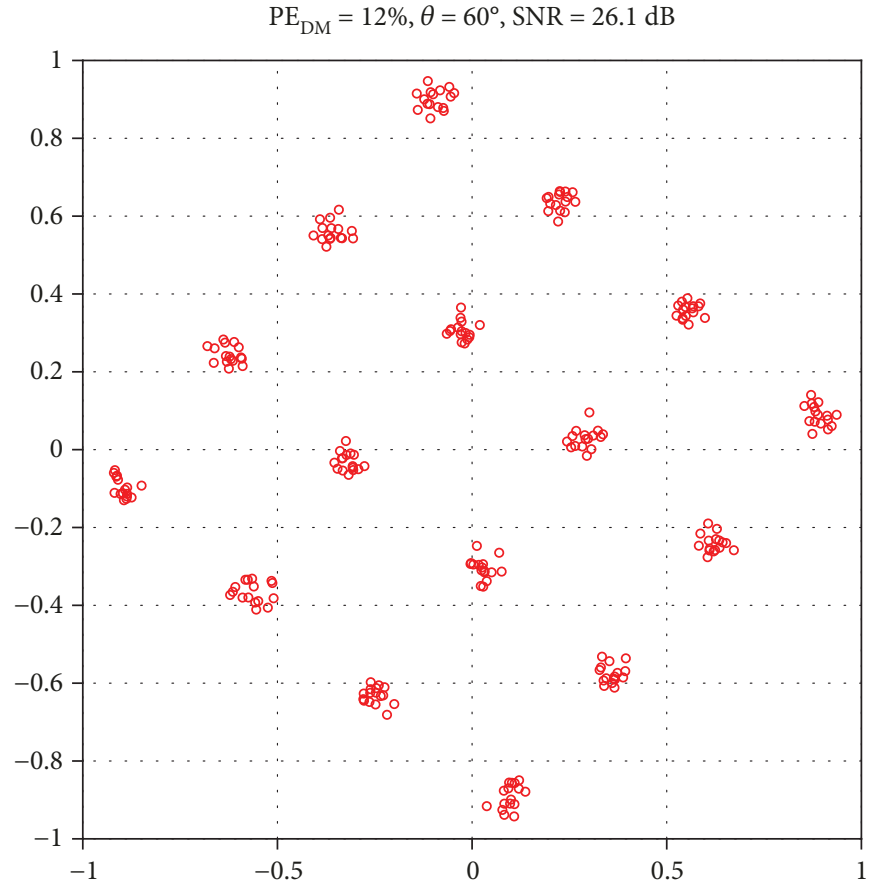

(b)

FIGURE 11: DDM 16-QAM modulation transmitted at the secure direction $60^{\circ}$ using real components, coaxial cables and (11) to compute $S_{21 \_m n}$ in the vector modulators; AEP measurement did not consider coaxial cables. (a) $\mathrm{PE}_{\mathrm{DM}}=50 \%$. (b) $\mathrm{PE}_{\mathrm{DM}}=12 \%$.

At first sight, one could think that, for these vector modulators, the unilateral assumption is reasonably well satisfied and the input and output are fairly well matched, so the use of (12) to compute $S_{21 \_m n}$ should be enough to generate similar radiation patterns to those that would be obtained with ideal components (Figure 7(a)). However, Figure 7(b) shows that the radiation patterns not only have less amplitude but also are slightly distorted.

As a result, the constellation generated at the desired secure direction of $60^{\circ}$ exhibits additional noise on the transmitted symbol (Figure 8). This behavior is due to the finite output return loss of the vector modulators $\left(S_{22_{n} n}\right)$ and the 


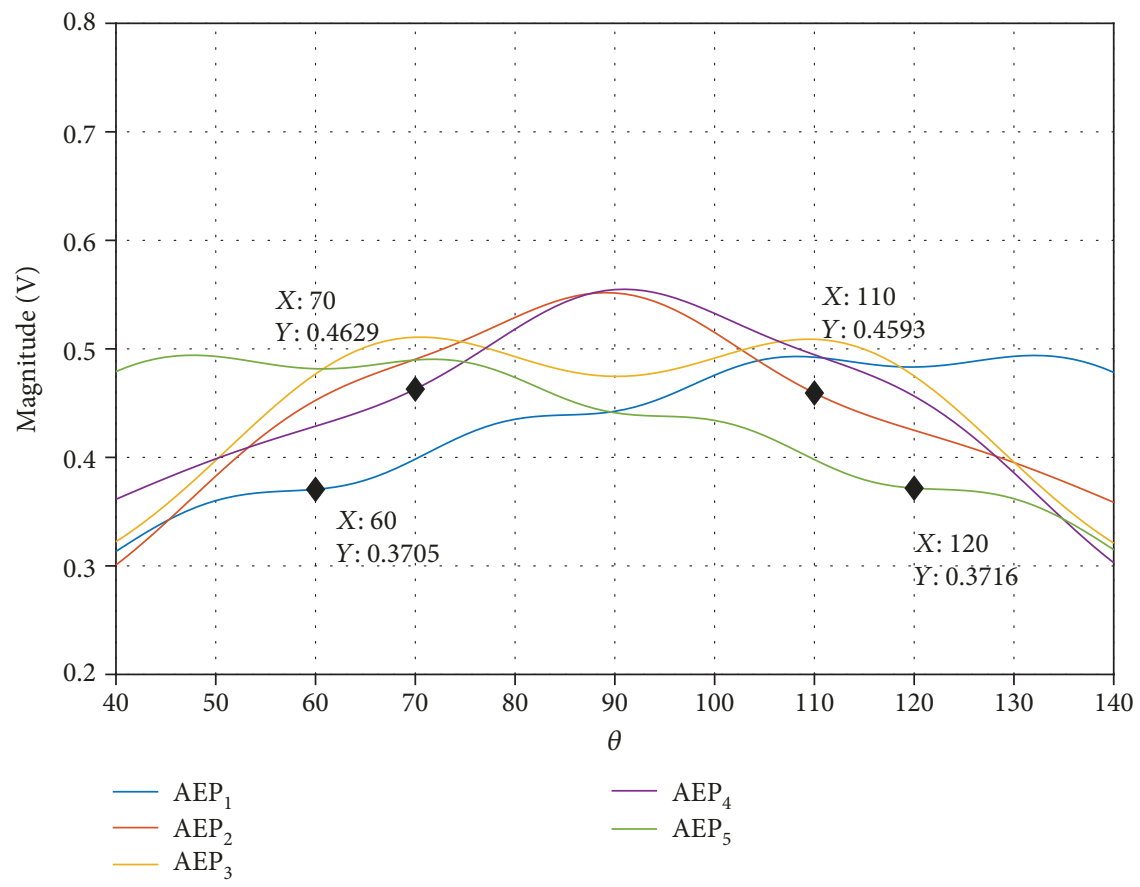

(a)

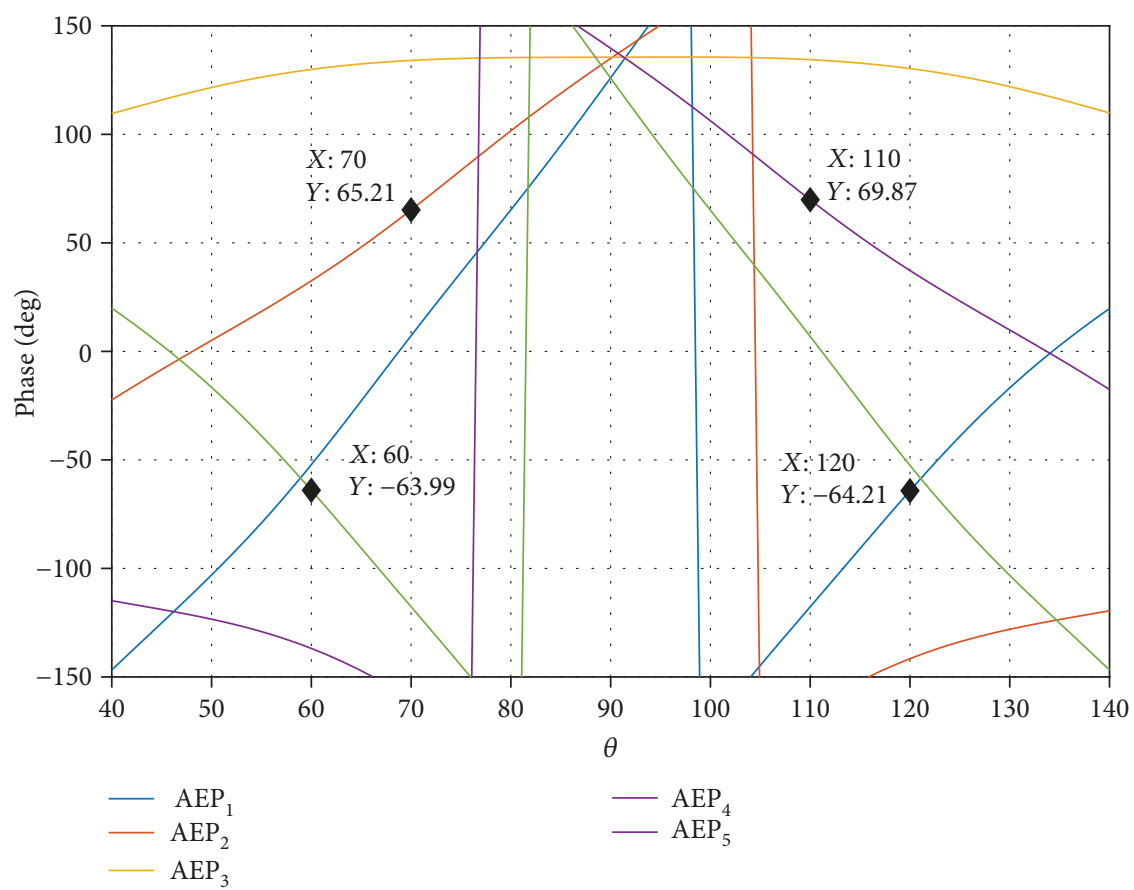

(b)

Figure 12: Active element pattern (H-plane) for an array of $5 \lambda / 2$ dipole antennas spaced $\lambda / 2$ with coaxial cables. (a) Magnitude. (b) Phase.

variation of the input reflection coefficient with the antenna and symbol to transmit $\left(\Gamma_{L_{-} m n}\right)$. In Figure 8, we have associated a signal-to-noise ratio (SNR) to the transmitted symbols that represents the threshold of quality that can be achieved in the transmission without additional calibration. Although SNR is still quite high for $\mathrm{PE}_{\mathrm{DM}}=50 \%$, it must be noted that the effects of real coaxial cables have not been considered here, so the case under consideration is quite favorable.
Another important result that can be derived from Figure 8 is that SNR decreases significantly with $\mathrm{PE}_{\mathrm{DM}}$. A low scenario $\mathrm{PE}_{\mathrm{DM}}$ would be required in the case of a highly secure transmission or in the case of simultaneous multichannel transmissions to different observation angles as in [13]. Therefore, these applications demand an accurate measurement of the components of the system and the use of (11) to obtain $S_{21 \_m n}$. Indeed, Figure 9 shows that, in this case, we 
can transmit the desired constellations without noise regardless of the value of $\mathrm{PE}_{\mathrm{DM}}$ used.

Finally, we add 5 commercial coaxial cables [20] in the system to connect antennas and vector modulators, which present attenuation of $1.1 \mathrm{~dB} \pm 0.1 \mathrm{~dB}$ and phase variation of $\pm 3^{\circ}$, and input and output return losses $\left(S_{i i}\right)$ ranged from 20 to $40 \mathrm{~dB}$.

Figure 10 shows the transmitted constellation for the secure direction of $60^{\circ}$ when we use (12) to compute $S_{21 \_m n}$, real components and coaxial cables which were not included in the AEP measurement. It is noticed that an improvement of over $3 \mathrm{~dB}$ in SNR with respect to Figure 8 is obtained. This result may seem counterintuitive since the addition of coaxial cables increases amplitude and phase variations. However, it makes sense if we consider that $\Gamma_{L_{-} m n}$ for the array with coaxial cables is not as sensitive to the symbol to be transmitted due to the additional losses. Conversely, as observed in Figure 10, we also see a reduction of $6 \mathrm{~dB}$ in the constellation amplitude which is significantly larger than the $1.1 \mathrm{~dB}$ introduced by coaxial cables.

Keeping the same scenario and using (11) instead of (12) to compute $S_{21 \_m n}$, we obtain some improvements (Figure 11). Now, the constellation amplitude has only been reduced by $1 \mathrm{~dB}$, as expected. Nevertheless, although SNR has improved 5-6 dB with respect to Figure 10, we still have noisy symbols. The only way to obtain the results of Figure 9 is by using the AEP of each element of the array with coaxial cables (Figure 12) in the computations. Notice in Figure 12 the additional attenuation of $1 \mathrm{~dB}$ and the slightly loss of symmetry with respect to Figure 5.

\section{Conclusions}

In this work, a FEKO model of a DDM system based on a power divider and vector modulators has been used to demonstrate that the system performance relies heavily on the precision with which the $S_{21}$ parameters of the vector modulators are set.

We have derived an expression for the $S_{21}$ parameters that takes into account the weight to be set for each antenna and symbol (that depends on the AEP of each antenna) and nonideal effects of the components such as unbalanced RF paths (amplitude and phase) and finite return losses.

Simulations show that, when nonideal effects are not considered in the computation of $S_{21}$, different sets of weights, that should generate the same symbol in a desired observation angle, produce slightly different symbols that have the appearance of noise. For that reason, we have associated a SNR to the transmitted symbols. This SNR decreases when we decrease $\mathrm{PE}_{\mathrm{DM}}$ to improve the security of the transmission in a single direction or to transmit, simultaneously, to different observation angles. Therefore, for these applications, the $S$ parameters of the components should be accurately measured and considered in the computation of $S_{21}$. Also, we have shown that the AEP of every antenna needs to be accurately evaluated together with the coaxial cables that feed the array. Small variations in amplitude and phase in the AEPs used to obtain the weights may result in a significant reduction of the amplitude of the transmitted symbols.

\section{Data Availability}

The FEKO model and data used to support the findings of this study are available from the corresponding author upon request.

\section{Conflicts of Interest}

The authors declare there is no conflict of interest regarding the publication of this article.

\section{Acknowledgments}

This work was supported by the Universitat Autònoma de Barcelona, the Spanish Ministry of Economy and Competitiveness, and FEDER funds through Project TEC201569229-R.

\section{References}

[1] A. Mukherjee, S. A. A. Fakoorian, J. Huang, and A. L. Swindlehurst, "Principles of physical layer security in multiuser wireless networks: a survey," IEEE Communications Surveys \& Tutorials, vol. 16, no. 3, pp. 1550-1573, 2014.

[2] S. A. Schelkunoff, "A mathematical theory of linear arrays," Bell System Technical Journal, vol. 22, no. 1, pp. 80-107, 1943.

[3] Y. Ding and V. F. Fusco, "A vector approach for the analysis and synthesis of directional modulation transmitters," IEEE Transactions on Antennas and Propagation, vol. 62, no. 1, pp. 361-370, 2014.

[4] Y. Ding and V. F. Fusco, "Establishing metrics for assessing the performance of directional modulation systems," IEEE Transactions on Antennas and Propagation, vol. 62, no. 5, pp. 2745-2755, 2014.

[5] Y. Ding, Y. Zhang, and V. Fusco, "Fourier Rotman lens enabled directional modulation transmitter," International Journal of Antennas and Propagation, vol. 2015, Article ID 285986, 13 pages, 2015.

[6] N. Valliappan, A. Lozano, and R. W. Heath, “Antenna subset modulation for secure millimeter-wave wireless communication," IEEE Transactions on Communications, vol. 61, no. 8, pp. 3231-3245, 2013.

[7] Q. Zhu, S. Yang, R. Yao, and Z. Nie, "Directional modulation based on 4-D antenna arrays," IEEE Transactions on Antennas and Propagation, vol. 62, no. 2, pp. 621-628, 2014.

[8] C. Sun, S. Yang, Y. Chen, J. Guo, S. Qu, and J. Hu, "4-D retrodirective antenna arrays for secure communication based on improved directional modulation," IEEE Transactions on Antennas and Propagation, vol. 66, no. 11, pp. 5926-5933, 2018.

[9] M. P. Daly and J. T. Bernhard, "Directional modulation technique for phased arrays," IEEE Transactions on Antennas and Propagation, vol. 57, no. 9, pp. 2633-2640, 2009.

[10] M. P. Daly, E. L. Daly, and J. T. Bernhard, "Demonstration of directional modulation using a phased array," IEEE Transactions on Antennas and Propagation, vol. 58, no. 5, pp. 15451550, 2010.

[11] H. Shi and A. Tennant, "Simultaneous multichannel spatially directive data transmission using direct antenna modulation," IEEE Transactions on Antennas and Propagation, vol. 62, no. 1, pp. 403-410, 2014. 
[12] Y. Ding and V. Fusco, "A review of directional modulation technology," International Journal of Microwave and Wireless Technologies, vol. 8, no. 7, pp. 981-993, 2016.

[13] S. Mufti, A. Tennant, and J. Parrón, "Dual channel broadcast using phase-only directional modulation system," in 2018 IEEE International Symposium on Antennas and Propagation (APS-URSI), pp. 2219-2220, Boston, MA, USA, 2018.

[14] E. A. Cabrera-Hernández, J. Parrón, and A. Tennant, "Influence of non-ideal components in a dynamic directional modulation system," in 2018 IEEE-APS Topical Conference on Antennas and Propagation in Wireless Communications (APWC), pp. 882-885, Cartagena de Indias, Colombia, 2018.

[15] FEKO EM, "Simulation software," https://altairhyperworks .com/product/FEKO.

[16] D. M. Pozar, "The active element pattern," IEEE Transactions on Antennas and Propagation, vol. 42, no. 8, pp. 1176-1178, 1994.

[17] D. M. Pozar, Microwave Engineering, John Wiley \& Sons, 4th edition, 2011.

[18] "5-way power divider and combiner (PS5 series)," February 2019, https://mcli.com/products/power-dividers-combiners/ stripline/5-way-power-divider-and-combiner-ps5-series.

[19] "HMC631 datasheet and product info analog devices," February 2019, https://www.analog.com/en/products/rf-microwave/ phase-shifters-vector-modulators/vector-modulators/hmc631 .html\#product-overview.

[20] “Coaxial cable RG174/U," February 2019, https://www. ecmstockroom.com/writable/items/pdf_files/rg174.pdf. 


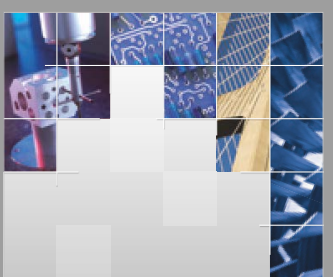

\section{Enfincering}
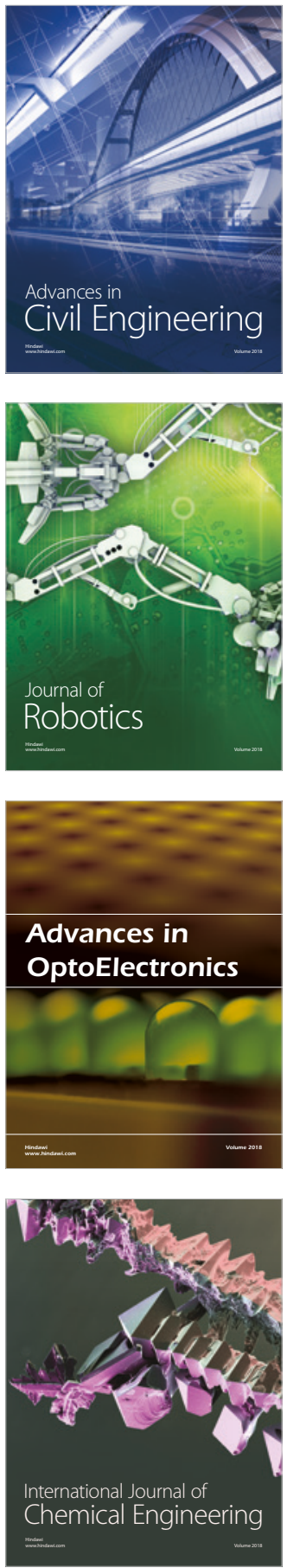

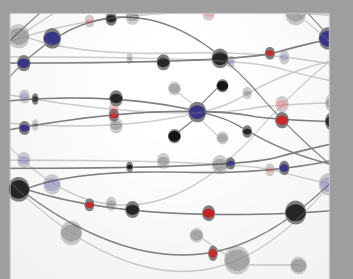

\section{Rotating \\ Machinery}

The Scientific World Journal

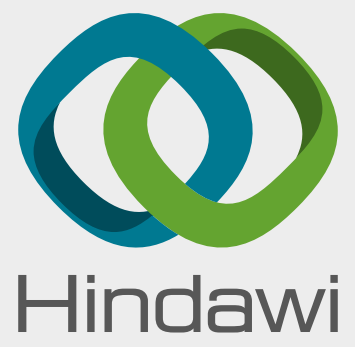

Submit your manuscripts at

www.hindawi.com
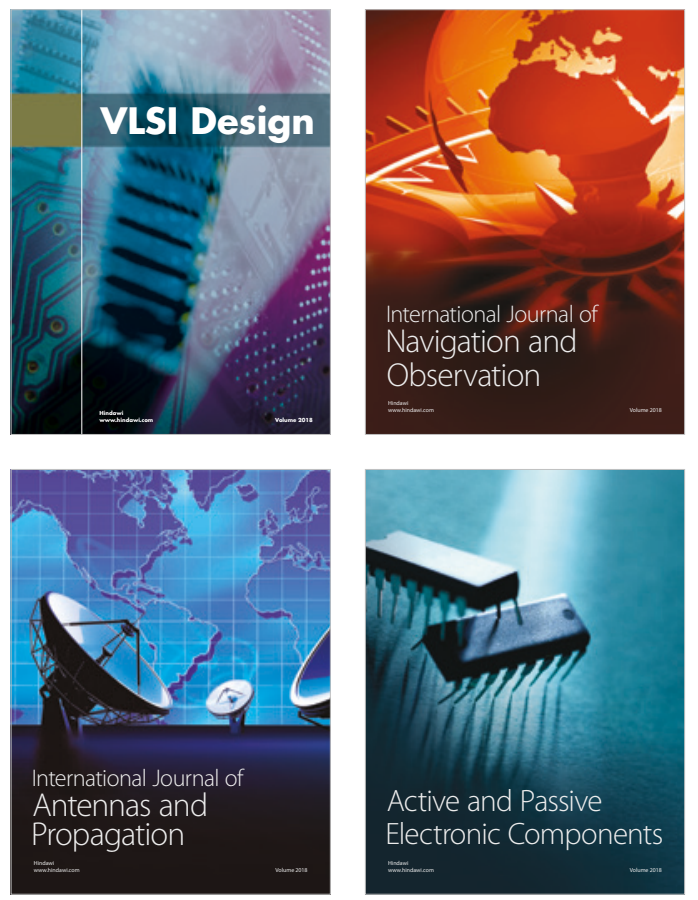
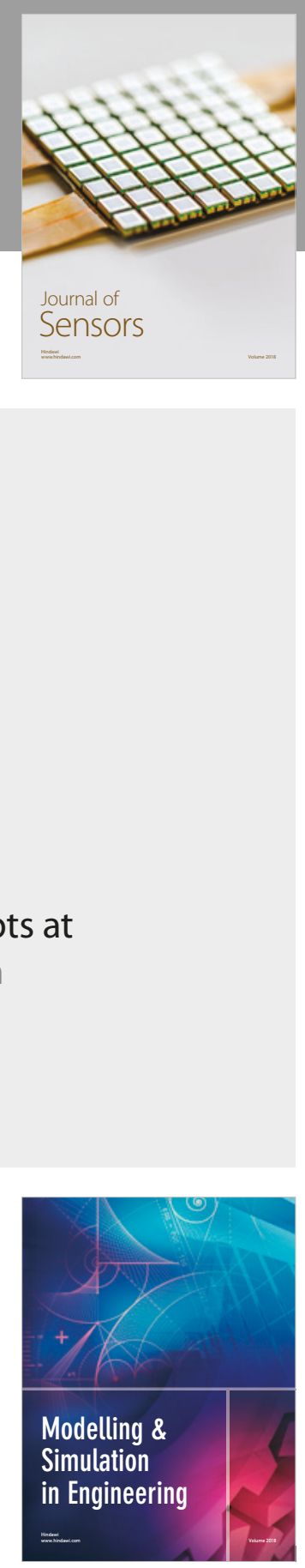

\section{Advances \\ Multimedia}
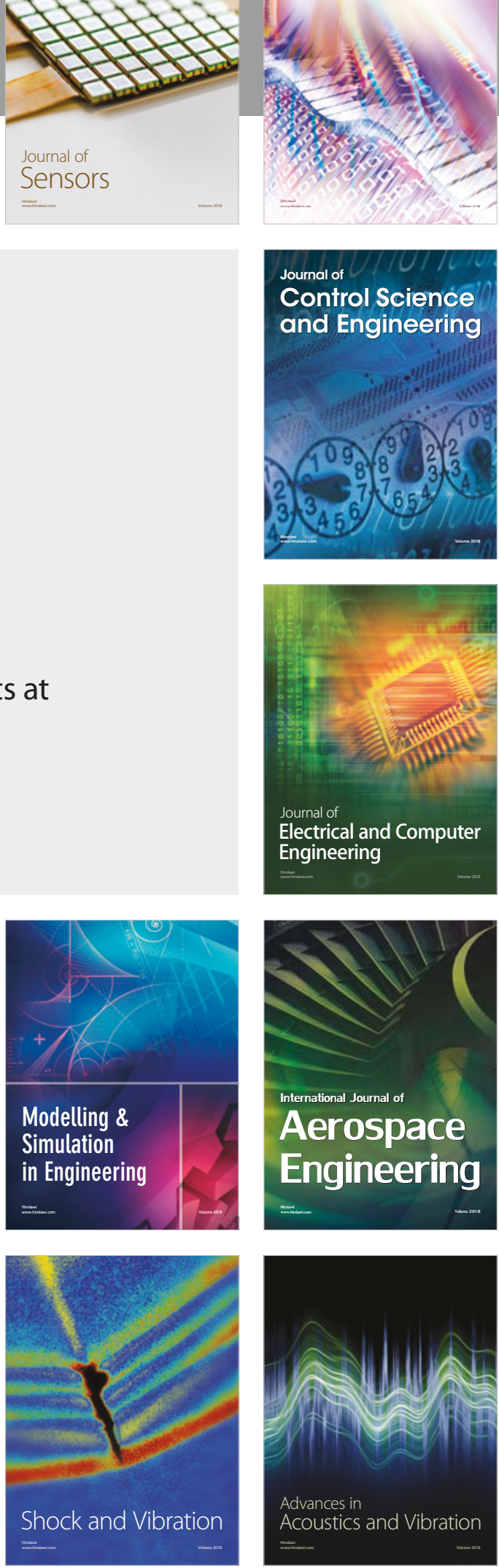